\title{
LOUIS ROUGIER ET LE NÉO-LIBÉRALISME DE L'ENTRE-DEUX-GUERRES
}

Au départ de l'itinéraire intellectuel de Louis Rougier se trouvent une analyse, une critique et un dépassement de la théorie de la connaissance. Il opère une axiomatisation de la pensée thomiste, retournant par là aux sources de ce qu'il nomme la "mentalité scolastique ». Il est un historien reconnu des religions. Ces deux disciplines, la philosophie et l'histoire des religions, constituèrent pour lui la matière d'un enseignement universitaire.

Ce ne fut point le cas - à tout le moins, pas d'une façon suivie et dans la longue durée d'une carrière - de sa réflexion d'économiste. Pourtant, cette réflexion n'est pas compréhensible si l'on ignore l'itinéraire intellectuel de Louis Rougier dans sa totalité : amené dans les années trente à construire une synthèse de ses analyses économiques, elle lui fut inspirée par l'ensemble de son œuvre antérieure.

Aussi importe-t-il de se pénétrer de la démarche de ce philosophe, historien, économiste, mais aussi politologue et sociologue, qui entendit appréhender la globalité du monde sensible, à la mesure de son intelligence critique. Avant de définir la pensée économique de Louis Rougier, telle qu'elle s'exprime au sein du courant néo-libéral de l'entre-deuxguerres, il semble nécessaire de tenir quelques propos liminaires sur le libéralisme classique et ses rapports avec une rationalité économique. Ces propos devront permettre de préciser, par la suite, les différences majeures entre les néo-libéraux et leurs prédécesseurs.

Ceux qu'on nomme les classiques, Adam Smith, John Stuart Mill, David Ricardo, ont, entre la fin du XVIII ${ }^{\mathrm{e}}$ siècle et le début du XIX ${ }^{\mathrm{e}}$ siècle, défini et démontré la supériorité du régime économique fondé sur l'intérêt personnel de l'individu et sur la concurrence. Leur analyse porte sur le mécanisme des prix et le fonctionnement des marchés, assurés par la loi de l'offre et de la demande, dans un cadre de liberté triplement fondamental : liberté de produire, liberté de vendre, liberté d'échanger. C'est la concurrence qui assure l'équilibre, le progrès et la souplesse d'un

Revue de synthèse: IV ${ }^{e}$ S. No 2, avril-juin 1989. 
système économique : l'équilibre, par l'égalité réalisée entre les quantités offertes et les quantités demandées; le progrès technique et économique, conséquent à l'amélioration des qualités et à la baisse des prix : la souplesse, enfin, car les producteurs concurrents doivent répondre aux variations de la demande.

L'existence de la concurrence était ainsi, pour les classiques, fondamentale, et ne justifiait pas qu'il existât une intervention de l'État; $a$ contrario, les forces vives de l'économie devaient être libérées des entraves à la libre production, à la libre circulation, au libre échange ${ }^{1}$.

L'économie a cependant connu entre la fin du $\mathrm{XIX}^{\mathrm{e}}$ siècle et le début du $X x^{e}$ siècle une double évolution. D'une part, on peut évoquer le développement croissant des cartels, trusts, unions de producteurs qui écartent la concurrence des marchés; d'autre part, c'est l'intervention de l'État dans l'économie. Cette intervention, manifeste pendant la Première Guerre mondiale, fut renforcée à la suite de la grande crise des années trente. Le système aboutit à une individualisation des bénéfices et à une socialisation des pertes, et suppose que l'État intervienne au-delà des fonctions traditionnelles que lui imposent les attributs de la souveraineté :

« [...] lieu central des divergences et des convergences d'intérêt, d'attentes et de comportements sociaux, il est, à tel moment, la seule instance qui doive et puisse intervenir. Quoique l'État (un appareil dont les administrations sont les bras séculiers) ait certainement une tendance "systémique " à s'étendre et à renforcer ses pouvoirs, il empiète sur les décisions privées et sur les mécanismes du marché surtout lorsque celles-ci ou ceux-ci sont défaillants, embarrassés ou en contradiction avec l'intérêt général ${ }^{2}$.

Ainsi, on peut comprendre que ce soit essentiellement autour du rôle de l'État dans l'économie que se constitue dans l'entre-deux-guerres en France la problématique d'une pensée néo-libérale, dans l'optique d'un "Retour au libéralisme " ${ }^{3}$. Si l'État doit jouer un rôle dans l'économie, c'est pour faire respecter la liberté économique, ce qui se situe aux antipodes de l'économie dirigée, mais qui n'est pas complètement

1. A cet égard, on peut rappeler l'cuvre essentielle de l'Assemblée constituante en France entre février et mars 1791 : en supprimant les douanes intérieures, les octrois, la gabelle, les aides, en février 1791, en interdisant coalitions, corporations, associations par la loi Le Chapelier du 14 mars 1791, elle abolit les entraves juridiques qui empêchaient que ne se réalisât une libération des emplois et de la production.

2. Le Capitalisme français: $19^{e}-20^{e}$ siècle, blocages et dynamismes d'une croissance, sous la dir. de Patrick Fridenson et André STrauss, Paris, Fayard, 1987, p. 31.

3. "Retour au libéralisme", titre d'un important article de Louis Rougier dans la Revue de Paris, paru le $1^{\mathrm{er}}$ janvier 1938. 
conforme à ce que Louis Rougier appelle la « mystique libérale », chère aux manchestériens, du "laisser-faire, laisser-passer " 4 .

L'on évoquera donc ce que les néo-libéraux nomment leur agenda (du latin : les choses qui doivent être faites) dans la France de l'immédiat avant-guerre, et dont Louis Rougier a réalisé dans ses écrits une synthèse $^{5}$. Éloigné du libéralisme classique, qui supposait que l'État n'intervînt pas dans le domaine économique, Louis Rougier attend, en effet, de l'État qu'il intervienne non politiquement, mais organiquement; il est bien plus éloigné encore du collectivisme ou du planisme - il semble chercher à définir les principes et les possibilités d'un État organique, d'un État situé entre le «tout à l'État " et le «tout à la société civile" qui serait fondé sur une solidarité organique de toutes ses composantes permettant de dépasser tous les types de clivages idéologiques; en quelque sorte un État sans politique ${ }^{6}$.

L'analyse de l'expression, par Louis Rougier, du néo-libéralisme français révèle trois domaines de réflexion spécifiques, qui concernent les activités de production et d'échanges, la monnaie, et la politique budgétaire de l'État. L'essai d'un bilan de la pensée néo-libérale et les directions de recherches qu'il peut susciter leur sont conséquents.

La réflexion des néo-libéraux à l'égard du domaine de la production se porte essentiellement sur les conditions de constitution - juridiques ou statutaires - des activités productives. Elle doit dans ce domaine beaucoup aux travaux de Walter Lippmann ${ }^{7}$. Ce journaliste et économiste américain, éditorialiste au New York Herald Tribune depuis 1931, était en correspondance avec Louis Rougier ${ }^{8}$. Les néo-libéraux mènent une critique fort sévère de la société anonyme par actions. Ils lui reprochent de dissocier la propriété des parts d'une entreprise de l'administra

4. L'expression de « mystique libérale " apparaît dans L. RougIER, Les Mystiques économiques. Comment l'on passe des démocraties libérales aux États totalitaires, Paris, Librairie de Médicis, 1938.

5. L'ensemble des documents concernant cet aspect de l'cuvre de Louis Rougier et se trouvant au château de Lourmarin a été classé par nous dans la section 8 du Fonds Rougier : Pensée et politique économiques.

6. Nous faisons ici référence à l'ouvrage de Michel Bouvier, L'État sans politique. Tradition et modernité, Paris, Librairie générale de droit et de jurisprudence, 1986.

7. L'étude qui suit se fonde sur les documents réunis dans la sous-section 8-3 (libéralisme économique) du Fonds Rougier du château de Lourmarin, qui contient en particulier les actes du Colloque Walter Lippman, tenu à Paris en 1938 sous les auspices de l'Institut international de coopération intellectuelle, et organisé par Louis Rougier. On se reportera aussi à Gaëtan Pirou, Néo-libéralisme, néo-corporatisme, néo-socialisme, Paris, Gallimard, 1939, et, du mème auteur, Les Nouveaux courants de la théorie économique aux États-Unis. T. 1 : Les Précurseurs, Paris, Domat-Montchrestien, 1939.

8. Ainsi qu'en témoignent les sous-sections 1.2 (correspondance générale) et 1.3 (correspondance : revues, congrès, comptes rendus) de la section 1 , Eléments de biographie, du Fonds Rougier. 
tion de celle-ci et, partant, font grief aux gestionnaires de chercher à priver les actionnaires d'une part des bénéfices. Cette attaque semble assez proche de celle qu'effectuent au même moment les catholiques sociaux dans les travaux des "Semaines sociales " et les socialistes de la S.F.I.O. Pour Walter Lippmann et Louis Rougier, la constitution de "holdings ", c'est-à-dire de sociétés de portefeuilles qui ne sont plus chargées de la gestion directe d'une entreprise, mais d'un regroupement d'actions issues de diverses sociétés, présente peut-être l'inconvénient le plus important : au sein de ces vastes systèmes financiers intégrés, la loi du marché finit par être exclue au profit de transactions financières qui ne dépendent plus d'elle. Ainsi se trouvent éliminées la concurrence et la liberté qui furent les conditions essentielles de l'essor du système économique au XIX $\mathrm{X}^{e}$ siècle. Ces critiques, au demeurant peu originales en 1938, présentent la spécificité de se poursuivre sur le terrain du politique puisque Walter Lippmann ajoute : « [...] de même que la Compagnie des Indes est devenue le gouvernement de l'Inde, de même les trusts géants, si on les laisse continuer, deviendront des départements des gouvernements " 9 .

Les néo-libéraux, devant l'évolution de ces grandes sociétés intégrées, qui vont jusqu'à préfigurer pour eux une gestion dirigée par une oligarchie des affaires, n'hésitent pas à faire intervenir l'État, dont le rôle serait alors d'interdire par la loi telle constitution d'entreprise ou telle méthode de gestion. Cet aspect de la réflexion néo-libérale présente un intérêt, dans la mesure où Lippmann et Rougier développent deux formes de cette intervention des pouvoirs publics, l'une ayant trait à l'autofinancement et l'autre aux conditions de l'échange.

L'autofinancement représente la part des investissements réalisée par les entreprises grâce à leurs propres ressources, une fois réalisés les amortissements nécessaires.

Il présente l'avantage de «[...] laisser à l'entreprise la plus large autonomie dans le choix de ses investissements, puisqu'elle les finance elle-même [...] cet autofinancement nécessite des marges de profit élevées $[\ldots] \gg{ }^{10}$. Or les néo-libéraux préconisent l'interdiction de l'autofinancement ${ }^{11}$. Une fois réalisés les amortissements et conservées les réserves de roulement, on contraindrait les sociétés à distribuer les bénéfices. Ce que veulent éviter les néo-libéraux, c'est de soustraire ces bénéfices à la loi du marché et à la libre concurrence, et d'aboutir à des

9. Walter Lippmann, La Cité libre, Paris, Librairie de Médicis, 1938, p. 263.

10. Jacques Garello, Jean-Yves NAUDET, L'Abécédaire de science économique, Paris, Tertiaire Conseils et Médica Gestion, 1985, p. 23.

11. C'est ce qui apparait à la lecture du dossier sur l'autofinancement contenu dans la sous-section 8.2 (politiques économiques) du Fonds Rougier. 
surinvestissements. La solution serait d'augmenter les salaires, soit de pratiquer une politique des revenus (ce qui revient à jouer sur la demande) et d'augmenter les dividendes des actionnaires. La capacité de consommation des uns et des autres s'en trouverait augmentée. On peut cependant faire deux remarques. Si l'autofinancement cesse, les investissements internes de l'entreprise s'arrêtent; il faut alors trouver des investissements auprès des banques. D'autre part, l'augmentation des revenus suscitée n'équivaut pas automatiquement à une augmentation des capacités productives des entreprises, et pourrait conduire à l'inflation et à la dépréciation monétaire. Cette critique de l'autofinancement, mode de financement efficace et non inflationniste, paraît ainsi peu fondée ${ }^{12}$.

Les conditions de l'échange font l'objet de propositions précises des néo-libéraux. L'acheteur ne dispose pas selon eux de la possibilité de juger de la qualité des marchandises qui lui sont offertes. Il est impuissant à vérifier, lorsqu'il réalise un achat, de la conformité du produit à la réclame. C'est alors aux pouvoirs publics qu'il appartient d'intervenir : ils doivent protéger le consommateur par un ensemble de mesures réglementaires. De même, l'État doit organiser certaines catégories de vendeurs, en particulier les paysans qui doivent vendre leurs produits sans connaître l'état de l'offre et de la demande. Ainsi dans ces deux domaines de la consommation et de la production, la formation de coopératives doit être le fait des pouvoirs publics.

Les questions monétaires font l'objet d'analyses approfondies des néolibéraux. On sait que les libéraux (classiques) considèrent la monnaie dirigée comme une manifestation dangereuse de l'économie dirigée. Dans l'immédiat avant-guerre, c'est en France l'étalon-or qui constitue la référence des économistes libéraux. Au premier rang de ceux-ci, il faut citer Jacques Rueff. Directeur adjoint puis directeur du Trésor, il joue un rôle à la fois technique et politique, proposant « [...] l'ajustement final et décisif des charges totales de l'État aux ressources qu'il peut effectivement mobiliser ${ }^{13}$. Il est un défenseur de l'étalon-or, ainsi qu'il l'exprime par exemple dans une conférence intitulée "Défense et illustration de l'étalon-or », lors du premier Congrès international d'écono-

12. D'autant que la France de l'immédiat avant-guerre ne dispose pas de capacités productives telles qu'une élévation des revenus les eût augmentées. Il ne suffit pas d'augmenter les revenus pour que la production suive; au contraire, l'offre joue un rôle moteur, ainsi que le disait au XIX $x^{e}$ siècle Jean-Baptiste Say, elle "crée sa propre demande".

13. Le Capitalisme français..., op. cit. supra n. 2, p. 47. On trouvera, dans le chap. II de cet ouvrage, une étude de la " ténébreuse autorité " de la haute administration publique du Trésor dans la France des années trente, dans le cadre des relations entre l'État et la croissance économique. La sous-section 8.1. (théorie monétaire) du Fonds Rougier contient par ailleurs un important dossier Jacques Rueff. 
mistes à Paris en 1937. Jacques Rueff et Louis Rougier se connaissent et s'estiment, ainsi qu'en font état leurs correspondances privée et professionnelle ${ }^{14}$. Walter Lippmann et Louis Rougier apportent certaines nuances, quant au rôle de la monnaie dans les transactions économiques, aux positions de l'orthodoxie libérale. Pour eux, c'est l'État qui doit assurer la loyauté de ces transactions économiques, en évitant que ne se modifie l'étalon de mesure des valeurs entre une prestation et une contreprestation. La monnaie est un intermédiaire des échanges, ainsi que l'affirmait Jean-Baptiste Say au début du $\mathrm{XIX}^{\mathrm{e}}$ siècle : " les produits s'échangent contre des produits ", ce qui signifie que la monnaie est neutre; elle « $[. .$.$] n'influence pas les grandeurs réelles (emploi, crois-$ sance) mais seulement les grandeurs nominales (inflation) ${ }^{15}$. Il importe donc de préserver le pouvoir d'achat représenté par la monnaie, ne varietur. Louis Rougier s'intéressait d'ailleurs à ces questions depuis des années : en 1920, l'année même où il obtint son doctorat ès lettres, il publiait dans la revue Organisation et Production un article dans lequel il reliait croissance monétaire et inflation ${ }^{16}$. Article inspiré par la conjoncture, dans la mesure où la hausse des prix avait dépassé en France les $200 \%$ en 1919 ; conscience de la fin d'une époque, celle de la stabilité des monnaies et des prix; réflexions sur les rapports entre les mouvements monétaires et les variables réelles qui préfigurent celles des monétaristes. Il ne semble pas inutile de rappeler, en effet, que dans les années trente, le système monétaire international rétabli lors de la Conférence de Gênes en 1922 n'existait plus. Les dévaluations successives de la livre sterling (1931), du dollar (1933) et, après des années de fidélité au bloc de l'or, du franc (1936-1938) avaient fait rentrer les monnaies des principaux pays développés dans des zones dangereuses de fluctuations erratiques, que les gouvernements tentaient de maîtriser. C'est pourquoi les néo-libéraux semblent accepter une intervention plus ferme des pouvoirs publics dans le domaine monétaire.

Le troisième aspect de la problématique néo-libérale concerne la politique budgétaire de l'État. Dans ce domaine, l'inspirateur des thèses néo-libérales semble avoir été Jacques Rueff - on note, en effet, une convergence certaine entre ses positions de 1936 à 1939 et d'importants aspects de la politique prônée par les néo-libéraux. Ces derniers s'accordent pour penser que la confiance publique - élément essentiel, variable non quantifiable de l'économie - ne pourra être restaurée que par un

14. Sous-sections 1.2 et 1.3. du Fonds Rougier.

15. J. Garello, J.-Y. NAUDET, op. cit. supra n. 10, p. 110.

16. L. Rougrer, "Le rôle de l'inflation monétaire dans la hausse des prix ", Organisation et Production, févr. 1920, p. 65-68. 
ajustement des dépenses de la nation à ses ressources. Cette politique de stricte orthodoxie budgétaire, proche d'un attachement à la déflation, n'est pas celle que réalise le gouvernement de Front populaire, puisque malgré l'ampleur du déficit ordinaire, la Chambre des députés vote en août 1936 un programme de grands travaux inscrit pour cinq milliards dans la loi de finances, au titre des charges extraordinaires civiles; en septembre de la même année, un programme de quatorze milliards de francs est voté au titre des charges extraordinaires militaires. Il apparaît que Rueff, directeur adjoint, puis directeur du Trésor a inspiré les décisions de la " pause » de mars 1937 qui réduit les grands travaux civils de deux milliards; il n'est pas étranger à la démission de Léon Blum du 21 juin 1937. Son scepticisme à l'égard d'une action positive de l'État en matière de relance économique l'entraîne à suggérer la conduite d'une politique économique plus libérale : "Dans la plupart des cas, on observe que les grands travaux d'État [...] se substituent purement et simplement à des travaux privés qu'ils rendent impossibles " ${ }^{17}$. La réduction des dépenses et le recours à une politique libérale sont nettement exprimés, ainsi que le recours au marché monétaire. La politique menée par Paul Reynaud au ministère des Finances de novembre 1938 à avril 1939 relève d'une logique voisine. La différence se situe dans la définition des ressources nouvelles en matière de fiscalité.

Dans ce domaine, la réflexion des néo-libéraux les entraîne vers des suggestions assurément novatrices. A la suite de Walter Lippmann, ils proposent des taxes de succession très fortes : l'argent est celui qu'on gagne et non celui qu'on garde. Il est curieux d'observer que Keynes parlait, à la même époque, d' " euthanasie fiscale du rentier ". De même, les impôts sur le revenu devront être rapidement progressifs. L'égalisation des revenus par l'impôt doit dans leur esprit servir à combattre le monopole, dans le cadre d'une fonction redistributrice des finances publiques. On peut objecter à ces vues qu'au-delà d'un certain seuil d'imposition, la hausse de la pression fiscale décourage l'effort productif, ce qui diminue ipso facto la masse du revenu imposable : l'impôt tue l'impôt. D'ailleurs, la politique économique de Paul Reynaud n'accrut que modérément et indirectement la pression fiscale, au contraire de ce qui est exposé ci-dessus.

L'analyse des thèses néo-libérales peut ainsi laisser la place à un essai de bilan et une indication des directions de recherches qu'elle suscite. Le néo-libéralisme a donc réussi dans trois domaines. La diffusion de ses idées, par le biais de livres, de périodiques, de colloques, en direction

17. Jacques RUEFF, Note du 16 juil. 1937, in Le Capitalisme frangais, op. cit. supra n. 2 , p. 54 . 
d'une opinion publique éclairée, a rencontré auprès de celle-ci une attention assez favorable. Témoin André Maurois qui écrit en 1938 :

"Avec le livre de Walter Lippmann, avec celui de Louis Rougier sur les Mystiques économiques, avec celui du professeur viennois Ludwig de Mises sur le Socialisme, nous assistons, en ces trois pays différents, à une renaissance intellectuelle du libéralisme. Cette renaissance est loin encore d'avoir atteint les masses, mais lorsque François de Chateaubriand écrivait le Génie $d u$ christianisme, le concordat de Bonaparte ne pouvait être bien loin. Quand une restauration est faite dans les esprits, il ne s'écoule jamais très longtemps avant qu'elle n'apparaisse dans les faits ${ }^{18}$.

André Maurois, perspicace observateur de son temps, a rétabli un lien fondamental de parenté intellectuelle et d'estime réciproque entre Louis Rougier et Ludwig von Mises; il faut de même remarquer que Louis Rougier fut un des premiers lecteurs de Friedrich von Hayek, un de ceux qui l'introduisirent en France et firent connaitre son œuvre.

Les raisons de ce succès auprès des élites intellectuelles tiennent sans doute à une différence notable des néo-libéraux, par rapport aux milieux conservateurs de la France de l'immédiat avant-guerre, ceux-là mêmes auxquels Alfred Sauvy reprochait une tendance au "malthusianisme économique ", et pour lesquels l'esprit de protection semble bien l'avoir emporté sur l'esprit de production. D'autre part, et Louis Rougier en est une illustration, on ne peut faire des néo-libéraux de simples porte-parole du patronat : certaines propositions en matière de fiscalité suffisent à le démontrer. Il est vrai que Louis Rougier a collaboré au Bulletin de la Société d'études des intérêts économiques, proche de la Confédération générale de la production française dans les années vingt. Il l'avait fait à la demande de celui qui supervisait la rédaction de ce Bulletin, et qu'il connaissait bien, André François-Poncet. Ce dernier, normalien et agrégé d'allemand, proche des milieux d'affaires et entré en politique après la Première Guerre mondiale avant d'être ambassadeur de France à Berlin de septembre 1931 à octobre 1938, puis à Rome jusqu'en juin 1940, permit à Louis Rougier de prendre une connaissance de la réalité concrète des milieux de l'entreprise; leurs relations étroites n'étaient d'ailleurs pas limitées au domaine de l'économie ${ }^{19}$. Ces remarques permettent d'établir l'existence d'une assez large diffusion des idées néolibérales dans des milieux divers qu'unissait une attention portée aux problèmes de leur temps.

18. André Maurois, préf. à W. LiPPMANN, op. cit. supra n. 9, p. 10.

19. Sous-sections $1.2,1.3$ et $1.4 \mathrm{du}$ Fonds Rougier. 
Le deuxième élément qui peut être porté au crédit des analyses néolibérales concerne leur dénonciation des systèmes d'économie dirigée. Louis Rougier s'est particulièrement attaché à mettre en cause le planisme économique, montrant qu'une économie intégralement planifiée ou purement collectiviste aboutissait à des effets contraires aux promesses qu'elle annonçait : le totalitarisme et l'abaissement de la dignité humaine, la militarisation de la population civile et la dégradation intellectuelle. Les raisons de ces échecs en sont l'impossibilité de tout calcul économique rationnel en économie planifiée, le développement de la bureaucratie, la perte de la (relative) liberté des agents économiques. Et Rougier de conforter ses analyses par une étude de la situation économique et politique de l'U.R.S.S., de l'Italie et de l'Allemagne de l'avant-guerre. Il écrit ainsi en juillet 1939 :

"Après avoir englouti l'Autriche, les Sudètes, la Tchécoslovaquie, le Moloch allemand cherche à convaincre les pays de l'Europe Centrale et du Sud-Est que le troc de leurs produits alimentaires et de leurs matières premières contre les marchandises allemandes doit être un processus obligatoire, non susceptible de révision, et l'Allemagne inscrit, d'ores et déjà, certains produits de ces pays dans son bilan de production, comme s'ils faisaient partie de son propre ravitaillement autonome. Le planisme, en engendrant la pénurie, puis la détresse économique, conduit donc les nations qui l'adoptent au militarisme agressif; et il force les autres nations à l'option suivante : ou se soumettre à l'hégémonie des États totalitaires ou affronter le risque de la guerre ${ }^{20}$.

Enfin, les positions du néo-libéralisme manifestent un attachement très marqué au libéralisme intellectuel et politique. Pour eux, les divers aspects de la liberté forment un tout, ce qui leur permet de penser qu'un pays dont l'économie ne serait pas libérale ne pourrait être un pays démocratique, puisque le système d'économie dirigée suppose un gouvernement qui fait plier sous la loi les intérêts particuliers, ce que seul un État totalitaire peut réaliser. Les néo-libéraux sont donc opposés au conformisme de pensée, à l'autoritarisme de gouvernement, aux totalitarismes, aussi bien communiste que fasciste :

" le glissement vers l'étatisme est interprété par les uns comme une évolution nécessaire inaugurant des temps nouveaux. A l'anarchie économique de l'État démo-libéral se substitue l'organicisme de l'État totalitaire [...] L'option n'est plus entre démocratie libérale et État autoritaire, mais entre ces

20. Le Planisme économique, ses promesses et ses résultats, exemplaire dactylographié, daté du 10 juillet 1939, in sous-section 1.6 du Fonds Rougier. 
deux formes de socialisme autoritaire : le fascisme et le communisme [...] deux formes de gouvernement et d'économie qui sont les espèces extrêmes d'un même genre ${ }^{21}$.

Les thèses néo-libérales, aussi brillamment exprimées qu'elles puissent être, n'en sont pas pour autant exemptes d'une triple réflexion critique. Les lois de 1863 et 1867 sur les sociétés anonymes par actions présentent effectivement des défauts dans l'ordre juridique et statutaire. Il semble, cependant, difficile de contester qu'elles ont pour l'essentiel permis le développement d'un système économique moderne dans la France du Second Empire ; en limitant la responsabilité de l'actionnaire, en rendant l'action simplement cessible, la société anonyme a permis de drainer l'épargne. Ce fut un des outils du système économique libéral.

Les ententes industrielles peuvent constituer des monopoles, et les critiques portées à cet égard par les néo-libéraux sont recevables. Toutefois, ces mêmes ententes présentent des aspects très positifs : discipline et rationalisation de la production, diminution des prix de revient, meilleure utilisation des machines et des matières premières, efficace solution aux problèmes des débouchés. Par ailleurs, l'entente est la conséquence de la concentration; la concentration est elle-même conséquente à la concurrence qui aboutit à l'élimination des entreprises peu productives. Il s'agit là d'un processus économique qu'on ne peut inverser par des mesures juridiques : le Sherman Act de 1890 aux États-Unis, plus connu sous le nom de loi antitrust en est un exemple. Enfin, la concentration des entreprises est un des aspects majeurs de l'efficacité du grand capitalisme du premier $\mathrm{XX}^{\mathrm{e}}$ siècle.

Quant à l'analyse, une remarque s'impose. Il est paradoxal de lire sous la plume de néo-libéraux français une dénonciation des ententes entre producteurs. Si aux États-Unis ou en Allemagne entre les deux guerres, et à un degré bien moindre en Grande-Bretagne, il existe de réelles concentrations d'industries, qu'elles soient horizontales ou verticales, la situation française apparaît fort différente. En 1931, on compte en France trente et un établissements de plus de 5000 salariés - on en comptait treize en 1896 - mais on dénombre 400000 établissements de moins de cinq salariés. Il n'existe dans la France des années trente que très peu d'entreprises modernes à l'américaine, et encore moins de concentrations industrielles; en revanche, on connaît un nombre significatif de « holdings $»$, ou sociétés de portefeuilles, phénomène révélateur de l'existence d'intégrations financières, semblables à des conseils d'administrations entrelacés, mais sans contrôle de la production et des ventes... ce que les

21. L. RovgIER, art. cit. supra n. 3, p. 2-4. 
néo-libéraux reprochent aux ententes ${ }^{22}$. Savoir quelle est la part de l'archaïsme et de la modernité dans la France d'avant la guerre, pour déterminer comment la croissance s'est nourrie à la fois des blocages et des dynamismes permet sans doute d'expliquer la spécificité de l'économie de notre pays et, partant, le paradoxe relevé plus haut des thèses néo-libérales.

Si l'on considère le lien qu'établissent les néo-libéraux entre le libéralisme économique et la démocratie, entre un système économique et un régime politique, tel que Louis Rougier l'affirma maintes fois dans son œuvre, les objections s'élevèrent dès le moment que furent énoncées ses positions. Une des plus remarquées fut celle de Marcel Déat. Ce normalien, agrégé de philosophie, avait quitté les socialistes de la S.F.I.O. en 1931 pour fonder un néo-socialisme qui entendait concilier des préoccupations sociales et d'évidentes sympathies pour le planisme, en rompant avec les origines idéologiques du socialisme. Adversaire déclaré du néolibéralisme, il qualifiait de "sophistique " l'assimilation du libéralisme économique au libéralisme politique, relevant que pour protéger la liberté et le fonctionnement correct de la démocratie, il fallait admettre que l'on pût limiter la liberté économique, dans le cas où celle-ci risquait de fausser les rouages de la démocratie ${ }^{23}$.

Cette assimilation, enfin, ne laissait pas de recevoir un démenti, dans le temps même qu'elle était affirmée. Dans la plus grande démocratie du monde, aux États-Unis, était pratiquée, depuis 1933 et l'arrivée à la présidence de Franklin D. Roosevelt, une politique économique qui visait à rendre au pouvoir politique une prééminence sur les milieux économiques, par l'intermédiaire du " New Deal », dont les perspectives sociales étaient également importantes. Les lois sur les syndicats, le second « New Deal » de 1938, après la double crise - politique et économique - de 1935 et 1937 montrent qu'on peut concilier un certain degré d'économie dirigée dans le cadre du système capitaliste (sans réformer les structures de l'économie) et une liberté politique, sans que cette remarque implique que l'on considère comme un succès la politique économique de l'administration Roosevelt.

Les néo-libéraux ne sont pas, enfin, exempts de discussions internes, et les analyses de ceux qui se réclament de leur mouvance peuvent révéler, dans l'immédiat avant-guerre des divergences, des incertitudes, des indéterminations. Les travaux du Colloque Walter Lippmann et les

22. Nous nous sommes fondé pour cette étude sur Richard KuISEL, Le Capitalisme et l'État en France. Modernisation et dirigisme au $X X^{e}$ siècle, Paris, Gallimard, 1984.

23. Sur les critiques du néo-libéralisme par Marcel Déat, on se référera à Marcel DÉAT et al., Néo-socialisme?, Paris, Grasset, 1933, et ID., «Conditions d'un équilibre français ", $X$-Crise, déc., 1938. 
conférences organisées par le Centre international pour la rénovation du libéralisme - ce dernier fonctionnant à partir de mars 1939, pour une assez courte période - révèlent, en effet, des échanges de vues constructifs mais aussi critiques ${ }^{24}$. Entre Walter Lippmann et les libéraux français, au sein même de ces derniers et malgré la synthèse souvent réussie par Louis Rougier, l'unanimité peut ne pas être évidente.

Ainsi les positions de Walter Lippmann concernant la neutralité de la monnaie ne semblent pas avoir convaincu l'ensemble des participants au Colloque, comme en témoignent les prises de position de Jacques Rueff ou de Louis Baudin. D'autre part, les concentrations industrielles sont dénoncées par Rueff qui considère à cette époque qu'une industrie cartellisée ou concentrée fait courir plus de risques à l'intérêt général qu'une industrie concurrentielle et disséminée, mais au prix d'une moindre efficacité économique. Une certaine indétermination se manifeste jusque dans la façon dont les Français proches de la mouvance néolibérale se nomment et qualifient leur courant de pensée. On a retenu dans cette étude le terme de néo-libéralisme, sans doute le plus exact, et le plus souvent employé. Louis Rougier employait également volontiers le terme de «libéralisme constructeur», pour différencier le néolibéralisme du libéralisme des classiques; on a évoqué un « libéralisme social " pour signifier que le capitalisme servait, mieux que le collectivisme ou le corporatisme l'intérêt du plus grand nombre; le néolibéralisme de l'avant-guerre fut souvent qualifié de «libéralisme rénové " par ses défenseurs.

En fait, ce sont deux grands courants de pensée qui doivent être dégagés d'une mouvance néo-libérale dans l'immédiat avant-guerre. Un courant foncièrement novateur, qui porterait au premier rang de ses thèses les devoirs de l'État dans les conditions économiques du capitalisme des années trente, et dans les conditions politiques de la démocratie contemporaine : illustré par Walter Lippmann, ce courant se devait de s'élargir aux couches populaires; il devenait, par ses aspects sociaux, plus proche d'un socialisme libéral que d'un libéralisme social, et la difficulté apparaissait très vite de la frontière entre ce courant et un interventionnisme qui insistait sur l'autorité plus que sur la liberté. Ce courant démontrait ainsi les écueils d'un individualisme social tendant au socialisme libéral.

L'autre courant, que l'on pourrait qualifier de modérateur, serait illustré par les néo-libéraux français tels que Rueff et Baudin. Dans la France des années 1938 et 1939, c'est ce courant qui semble avoir

24. Sous-section $8.3 \mathrm{du}$. Fonds Rougier. 
prédominé chez les néo-libéraux, en convergence avec la politique économique de Paul Reynaud qui, refusant tout contrôle de l'État sur l'économie, déclarait néanmoins que sa politique s'inscrivait dans le sens d'une "... économie guidée dans le cadre de la liberté»" ${ }^{25}$. Louis Rougier a, par ses écrits, réalisé fréquemment un point de convergence entre les deux courants; s'il retient à plusieurs reprises les thèses de Walter Lippmann, c'est du côté des néo-libéraux français qu'il semble possible de le situer convenablement, même s'il a souvent exprimé des opinions qu'il faisait siennes, et sans toujours pouvoir préciser ce qui lui revient avec évidence.

Au terme de cette étude demeurent deux éléments fondamentaux, qui font du néo-libéralisme français de l'avant-guerre un courant de pensée important, auquel Louis Rougier a contribué.

D'une part, le néo-libéralisme affirme la responsabilité individuelle des agents économiques et fonde par là même le système d'économie de marché en devenir. L'intérêt personnel, l'initiative privée, les diversités individuelles sont reconnus, même si l'on a remarqué que Louis Rougier pouvait minimiser les capacités d'un individu dans le cadre de ses comportements micro-économiques. Dans le même esprit et conséquemment, il démontre avec force ce que comportent de chimérique les projets de planification intégrale et les systèmes qui substituent au comportement individuel une " mystique » du service social.

D'autre part, il insiste sur l'existence de lois économiques, en distinguant deux catégories d'intervention de l'État ${ }^{26}$. Les premières sont en contradiction avec les mécanismes qui sont à la base du fonctionnement du système économique, c'est-à-dire les mécanismes des prix. Contrevenant aux conditions de l'équilibre économique, elles engendrent de graves perturbations; la législation sur les prix des biens et des services en est l'exemple flagrant. Les secondes ont intégré le fonctionnement des mécanismes économiques, leurs actions et leurs réactions respectives, et s'efforcent de les infléchir dans un sens conforme à un objectif déterminé. Une action indirecte sur les prix pourrait, dans ces conditions, être envisagée, par une politique de l'offre engagée par la volonté des pouvoirs publics. C'est reconnaître par là que l'État peut intervenir dans un sens a-économique dans le domaine de l'économie.

C'est dans cette seconde perspective que se situe le néo-libéralisme

25. Paul ReYNaud, Mémoires, p. 238, cité par R. Kuisel, op. cit. supra n. 22, p. 224.

26. Nous nous sommes fondé pour ce qui suit sur une conférence prononcée par J. RUEFF au Céntre international d'études pour la rénovation du libéralisme le 13 mars 1939 , qui se trouve dans le Fonds Rougier en 8, Pensée et politique économiques. G. PIROU en avait fait une analyse in Néo-libéralisme, néo-corporatisme, néo-socialisme, op. cit. supra $\mathrm{n} .7$, p. 67-69. 
français de l'immédiat avant-guerre, auquel Louis Rougier appartint éminemment. Il admet que les valeurs politiques puissent primer les valeurs économiques. Adam Smith, qui pensait que «... personne ne tolère la totale liberté du marché ", reconnaissait également que «... la sécurité prime la richesse ». Cette réintroduction de l'éminence du politique sans la politique, du gouvernement de la Cité sans les idéologies, fait du néo-libéralisme de Louis Rougier un courant, inscrit dans une école de pensée dont les écrits et les actes marquèrent l'immédiat avantguerre français. Elle connut, de façon récente, une postérité tardive. Si "le propre du sage est d'ordonner", donnons la parole à Louis Rougier :

"La science économique nous révèle ainsi qu'il est vain de chercher à sauvegarder des institutions démocratiques, en recourant aux méthodes du dirigisme autoritaire. Elle établit que le socialisme libéral, dont rêvent tant de républicains sincères, est une contradiction dans les termes. Le socialisme est autoritaire ou il n'est pas, et l'on ne peut défendre les Droits de l'homme et du citoyen tout en planifiant l'économie. Libre à chacun de préférer Sparte à Athènes, le totalitarisme au libéralisme, le communisme au capitalisme; mais on ne peut réaliser la conciliation des contradictions. On ne peut introduire des réformes de structure dans un régime capitaliste, sans lui faire perdre son ressort - un État totalitaire ne peut devenir libéral sans renoncer à lui-même; Athènes, au temps de sa splendeur, eût pu adopter la discipline militaire de la rigide Sparte, mais elle eût cessé d'être la cité libre, l'école de la Grèce, l'institutrice du genre humain ${ }^{27}$.

Ainsi apparaît une objection dernière et sans doute décisive, au-delà de celles soulevées plus haut et qui s'apparentent à une critique libérale et économiste de la pensée de Louis Rougier. La réalité objective de l'État révèle son incapacité à ne jouer qu'un rôle technique dépourvu de finalité, ce vers quoi Rougier semble tendre en 1939. Cette réalité est indissociable d'une pensée du pouvoir, qui n'est pas explicite dans les constructions intellectuelles que nous avons présentées.

La portée de cette ultime réserve est à la mesure de l'intérêt de la pensée de Louis Rougier : ni le seul État, ni le seul marché, elle préconise une troisième voie, une régulation intermédiaire, organique, par le biais de groupes fonctionnels, sans exclure ni le rôle du premier ni celui du second. Mais quant à savoir où se situerait la réalité du pouvoir, qui assurerait l'ordre contre le désordre, (ou la qualité contre le nombre?), le problème reste entier et dépasse la problématique des rapports entre Louis Rougier et le néo-libéralisme français de l'avant-guerre, tant il est

27. L. RoUgIER, op. cit. supra n. 4, p. 196. 
vrai qu' "il ne dépend pas seulement de rapprocher les individus du pouvoir par le biais des corps intermédiaires pour que soit garantie la liberté de chacun ${ }^{28}$.

Tristan LECOQ, École supérieure de commerce Le Havre-Caen.

28. M. Bouvier, op. cit. supra n. 6, p. 244. 